Measurements of heavy-ion anisotropy and dose rates in the Russian section of the International Space Station with the Sileye-3/Alteino detector

This content has been downloaded from IOPscience. Please scroll down to see the full text. 2015 J. Phys. G: Nucl. Part. Phys. 42025002

(http://iopscience.iop.org/0954-3899/42/2/025002)

View the table of contents for this issue, or go to the journal homepage for more

Download details:

This content was downloaded by: oscarlar IP Address: 134.160.214.28

This content was downloaded on 15/12/2014 at 00:44

Please note that terms and conditions apply. 


\title{
Measurements of heavy-ion anisotropy and dose rates in the Russian section of the International Space Station with the Sileye- 3/Alteino detector
}

\author{
O Larsson ${ }^{1}$, VV Benghin ${ }^{2}$, T Berger ${ }^{3}$, M Casolino ${ }^{4,5}$, \\ L Di Fino ${ }^{4,6}$, C Fuglesang ${ }^{1}$, M Larosa ${ }^{3,6}$, B Lund-Jensen ${ }^{1}$, \\ A Nagamatsu ${ }^{7}$, L Narici ${ }^{4}, 6$, IV Nikolaev ${ }^{2}$, P Picozza $^{4,6}$, \\ G Reitz ${ }^{3}$, C De Santis ${ }^{4,6}$ and V Zaconte ${ }^{4,6}$ \\ ${ }^{1}$ Royal Institute of Technology, Albanova University Center, SE-10691, Stockholm, \\ Sweden \\ ${ }^{2}$ Institute for Biomedical Problems, Moscow, Russia \\ ${ }^{3}$ German Aerospace Center, Köln, Germany \\ ${ }^{4}$ INFN sect. Roma Tor Vergata, Rome, Italy \\ ${ }^{5}$ RIKEN, Wako, Saitama, Japan \\ ${ }^{6}$ University of Rome Tor Vergata, Rome, Italy \\ ${ }^{7}$ Japan Aerospace Exploration Agency, Japan \\ E-mail: OscarLar@KTH.se
}

Received 15 June 2014, revised 4 October 2014

Accepted for publication 7 October 2014

Published 11 December 2014

\begin{abstract}
In this work we present data on linear energy transfer (LET), dose and dose equivalent rates from different locations of the Russian part of the International Space Station (ISS) measured by the Sileye-3/Alteino detector. Data were taken as part of the ESA ALTCRISS project from late 2005 through 2007. The LET rate data shows a heavy-ion (LET $>50 \mathrm{keV} / \mu \mathrm{m}$ ) anisotropy. From the heavy-ion LET rate in the Zvezda service module we find ISS $\hat{y}$ (Starboard) and $\hat{z}$ (Nadir) to be about 10-15 times higher than in $\hat{x}$ (Forward). The situation is similar for dose and dose equivalent rates, ranging from $25-40 \mu \mathrm{Gy} \mathrm{d}^{-1}$ in $\hat{x}$ to about $75 \mu \mathrm{Gy} \mathrm{d}^{-1}$ in $\hat{z}$, whereas for the dose equivalent the rate peaks in $\hat{y}$ with around $470 \mu \mathrm{Sv} \mathrm{d}^{-1}$. The heavy-ion anisotropy confirms what has been reported by the ALTEA collaboration. Measurements using two sets of passive detectors, DLR-TLDs and PADLES (TLD+CR-39), have also been performed in conjunction with Alteino measurements, both shielded and unshielded. The passive detectors register a dose rate about 3-5 times as high as Alteino, 260-280 $\mu \mathrm{Gy} \mathrm{d}^{-1}$ for PADLES and 200-260 $\mu \mathrm{Gy} \mathrm{d}^{-1}$
\end{abstract}


for DLR-TLDs. For the dose equivalent PADLES measurements ranges from $560-740 \mu \mathrm{Sv} \mathrm{d}^{-1}$.

Keywords: Alteino, ISS, dose, dose equivalent, LET, heavy ion

(Some figures may appear in colour only in the online journal)

\section{Introduction}

The International Space Station (ISS) is a unique and valuable platform in preparation of continued human exploration of space. One of the challenges is to find an effective way of protecting the crew on a long duration deep space mission from cosmic radiation. As part of this ongoing effort, the radiation environment on-board the ISS is monitored by several detectors, both passive and active with different spectral response profiles. The cosmic ray flux in the orbit of the ISS is modulated by geomagnetic influence, solar activity, and the inhomogeneity in the hull and placements of racks and other equipment inside the ISS. Therefore it is still an open question to assess, a priori, the cosmic ray flux and hence, the corresponding dose, and dose equivalent rates in different places inside the Space Station [1-8].

The Sileye-3/Alteino particle detector measures the cosmic ray particle flux and composition inside the ISS. It follows Sileye and Sileye-2, which both flew on the Russian space station Mir in the 1990s [9-13]. Alteino was launched in 2002 and placed in the Pirs module. During the ESA ALTCRISS project (Alteino long term monitoring of cosmic rays on the ISS) [14], measurements started in the Pirs module in late December 2005. In early 2006 the detector was moved into the Russian service module, Zvezda, and several measurements in varying locations, orientations and configurations were performed over a period of 30 months. The data measured inside the ISS will be useful for comparison of radiation levels inside and outside the ISS. This in turn will be useful for estimations of astronaut dose rates on future space missions to the Moon or Mars.

In this report we present data on the heavy-ion anisotropy in the three orthogonal orientations of the ISS, measured in the Zvezda service module. In addition to LET rate $\left(\mathrm{LET}_{r}\right)$ we report on dose and dose equivalent rates ( $D$ and $H$ respectively).

\section{Detector description}

The Alteino particle detector $[15,16]$ consists of eight silicon strip detector planes, each with a thickness of $380 \mu \mathrm{m}$. Each plane is 8 by $8 \mathrm{~cm}^{2}$ with a strip pitch of $2.5 \mathrm{~mm}$, totalling 32 strips per plane. Planes are stacked with strip orientation alternating in $\hat{X}$ and $\hat{Y}$ (detector frame of reference), enabling track reconstruction by calculating change in $\hat{X}$ and $\hat{Y}$ positions through the detector. The read-out is triggered by a logic AND signal from two scintillators, one on the top and one on the bottom of the silicon plane stack, each $1 \mathrm{~mm}$ thick. The detector is however not capable of directional discrimination, i.e. $\pm \hat{Z}$ of the particles momentum vector in the detector frame of reference. The detector geometry and trigger configuration selects particles with kinetic energy above $\approx 60 \mathrm{MeV} / \mathrm{n}$ for carbon and above $\approx 126 \mathrm{MeV} / \mathrm{n}$ for iron nuclei [17]. In this work we focus on the heavy-ion range, thus, the trigger threshold which discriminate against lighter nuclei (protons to beryllium) is not a concern. However, even using a high trigger threshold, a large portion of light nuclei will still trigger the readout. 
Table 1. Summary of the data sets analysed in this report. 'Direction' refers to which orientation the stacking of Alteino is facing in respect to the ISS coordinate system. 'Loc.' is where Alteino was placed ('SM-\#' refers to Zvezda module and panel number and 'P-\#' to Pirs module and panel number). 'PE' if polyethylene is used. 'Time' indicates starting date and duration of measurement.

\begin{tabular}{llccc}
\hline $\begin{array}{l}\text { Data } \\
\text { Set }\end{array}$ & Loc. & Direction & PE & $\begin{array}{c}\text { Time } \\
\text { [yy-mm-dd + dd] }\end{array}$ \\
\hline 924 & SM-437 & $\hat{x}$ & Yes & $06-11-10+3.79$ \\
918 & SM-437 & $\hat{x}$ & Yes & $07-01-18+13.3$ \\
925 & SM-326 & $\hat{x}$ & No & $07-05-22+17.2$ \\
\hline 906 & SM-239 & $\hat{y}$ & Yes & $06-02-26+15.1$ \\
907 & SM-239 & $\hat{y}$ & Yes & $06-03-16+21.1$ \\
\hline 910 & SM-333 & $\hat{z}$ & Yes & $06-05-17+9.31$ \\
930 & SM-326 & $\hat{z}$ & Yes & $07-06-27+21.0$ \\
\hline 902 & P-401 & $\hat{x y}$ & No & $05-12-26+11.2$ \\
903 & P-401 & $\hat{x y}$ & Yes & $06-01-08+15.4$ \\
\hline
\end{tabular}

In some sessions extra shielding material $\left(5 \mathrm{~g} \mathrm{~cm}^{-2}\right.$ Polyethylene pads, PE) were placed infront of the acceptance windows on both sides of the detector to evaluate the materials shielding capability in respect to cosmic heavy ions.

During most of ALTCRISS project measurements passive Thermoluminescent detectors (TLDs [18]) and plastic nuclear track detector (CR-39 detectors [19]) were used in conjunction with Alteino [14]. DLR ${ }^{8}$ supplied pairs of TLDs, each pair containing TLD600 and TLD700 of the same types as in the Dosemap experiment [2]. In addition, PADLES pouches, containing both TLD and CR-39(TD-1 type) detectors, were supplied by Japanese JAXA $[3,20,21]$. These pouches were placed in the same configuration as the DLR-TLDs. Two pairs were constantly shielded, and one pair was unshielded. When the PE was mounted on Alteino, the shielded dosimeters were sandwiched between the PE and the detector window, and when not mounted, the PE pads were fixed on top of each other with the dosimeters in between. In this work we present passive dosimeter data from the initial phase of the ALTCRISS experiment (Dec '05-April'06).

\section{Data analysis}

The detector response and calibration have previously been investigated $[16,22]$. The stability of the linearity for $\mathrm{d} E / \mathrm{d} x$ versus $z^{2}$ over time has been shown and a conversion factor of $3.3 \mathrm{keV} / \mathrm{ADC}$ has been reported. Over the years the detection efficiency in some strips decreased, therefore to measure the LET we have applied detector geometry cut, selecting a core region with the highest efficiency. Analytical calculations using formulae from [23] in combination with Monte Carlo simulations using Geant4 [24, 25] have been performed to reevaluate the geometrical factor for the selected detector geometry. The efficiency has been evaluated for each strip $(i)$ in the fifth layer using coincidence hits in the corresponding strip $i$ in first and seventh layers. The average plane efficiency was evaluated to $82 \%$ with an estimated systematic error of $4 \%$ for all ALTCRISS data sets. The efficiency has been

${ }^{8}$ German Aerospace Center. 


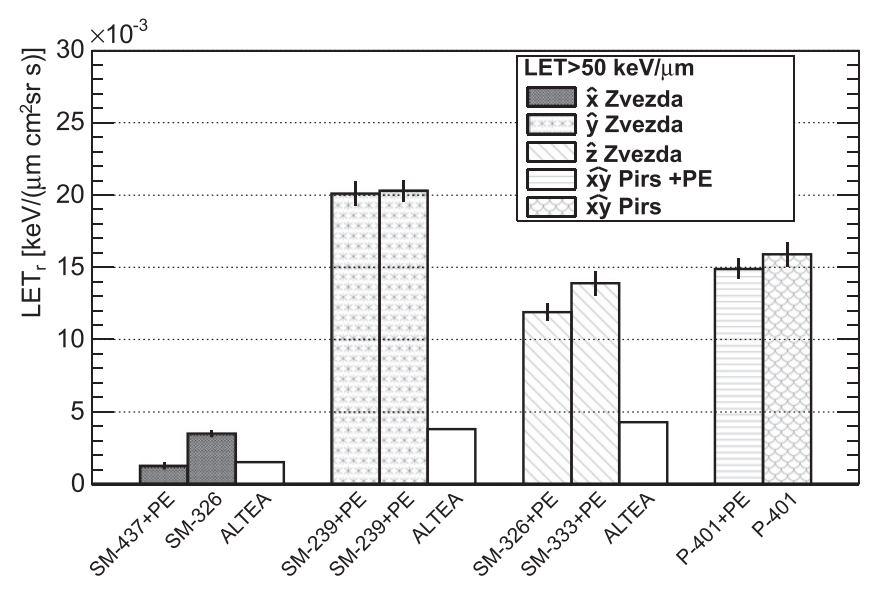

Figure 1. The heavy ion LET rate in the three orthogonal directions from the Zvezda module together with ALTEA measurements from the Destiny module [6]. To the right the two Pirs measurements where the P-401+PE is with the $5 \mathrm{~g} \mathrm{~cm}^{-2} \mathrm{PE}$ shield. Statistical error for Alteino is $<1 \%$, and for ALTEA; $0.01 \%$.

estimated for the whole detector. However, only data from the fifth layer have been selected since it showed the highest efficiency of all planes. In addition, small discrepancies, within the statistical uncertainties, in the efficiency between different layers were observed. A summary of the data sets is presented in table 1 . The ISS axes are $+\hat{x}$ : forward tangent of the orbit, $+\hat{y}$ : starboard, and $+\hat{z}:$ is nadir. The $\hat{x y}$ notation refers to Alteino's silicon planes being at a 45 degree angle to both $\hat{x}$ and $\hat{y}$ and parallel to the $\hat{z}$ axis.

Further event selection was based on incident angle and strip multiplicity. A few events that show a very high multiplicity $(90 \%$ of the strips register hits in the detector for a single event) due to electronic noise are rejected. In addition, if the traversing angle can not be determined, the energy deposit per track length can not be deduced and the event is rejected.

To evaluate the dose rate, the LET in silicon $\left(\mathrm{LET}_{\mathrm{Si}}\right)$ has been converted to LET in water $\left(\mathrm{LET}_{\mathrm{w}}\right)$ using the relation from [26] and subsequently to dose equivalent by a quality factor defined by ICRP [27]. The average $Q$-value $(\langle Q\rangle)$ reported here was calculated as $H / D$.

\section{Results and discussions}

The lower bound for the total LET rate $\left(\mathrm{LET}_{r}=\int \Phi \cdot \mathrm{dLET}\right)$ calculation was set to $\mathrm{LET}_{\mathrm{Si}}$ $>3 \mathrm{keV} / \mu \mathrm{m}$. For heavy-ion $\mathrm{LET}_{r}$ the lower bound was set to $\mathrm{LET}_{\mathrm{Si}}>50 \mathrm{keV} / \mu \mathrm{m}$, corresponding to relativistic magnesium and above. The Alteino data, measured in the Zvezda module, presents a clear anisotropy in the $\mathrm{LET}_{r}$ for both total and heavy-ion rates in $\hat{x}$ compared to $\hat{y}$ and $\hat{z}$. The data also indicate a clear difference in heavy-ion $\mathrm{LET}_{r}$ in $\hat{z}$ when compared to $\hat{y}$. These differences in rates can be understood by the difference in shielding. When looking in the $\hat{x}$-direction the main body of the ISS is in the field of view and the total shielding is at its maximum. In $\hat{z}$ we expect shielding contribution from the hull and racks in both directions. In addition, the Earth will be shielding a portion of the impinging flux and thus we expect lower rates. Whereas in $\hat{y}$ one would expect similar hull and rack configuration as in $\hat{z}$ to contribute to the shielding, though no additional shielding from the Earth. However, in our $\hat{y}$ measurements half of the presumed rack shielding is missing since the detector was placed next to the hull in the port side crew cabin. The difference in the heavy 


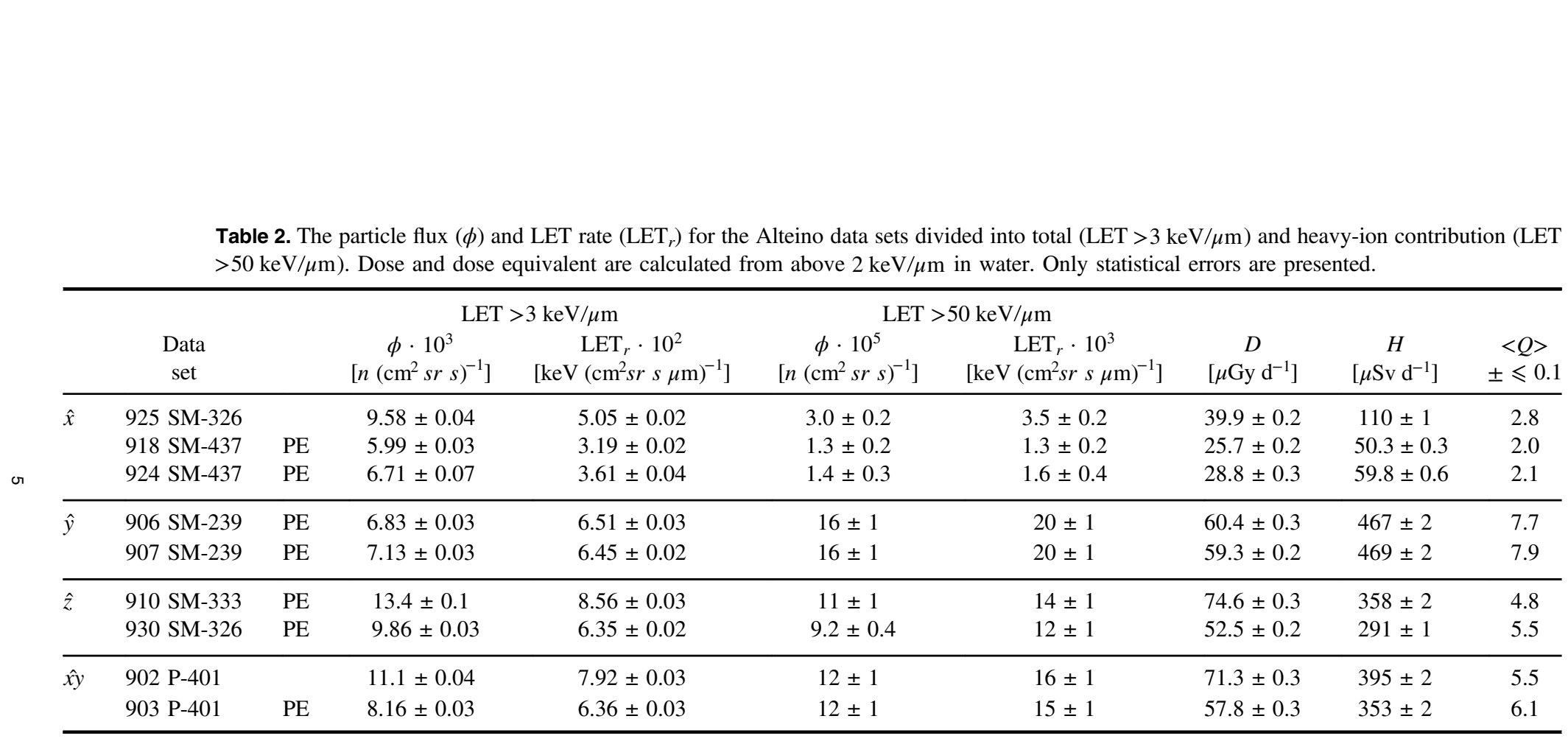




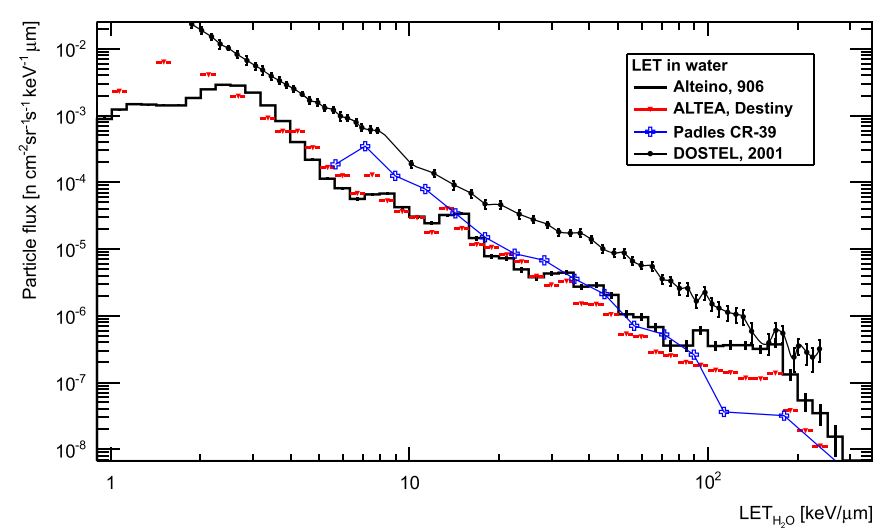

Figure 2. The LET equivalent response in water for Alteino, data set 906 (solid histogram), PADLES measurement without shielding (blue hollow crosses), and for comparison two measurements from the Destiny module: DOSTEL (black connected circles) measured in 2001 [2] reproduced with the permission of Oxford University Press, and ALTEA (red diamonds) measured in 2009 [6].

ion $\mathrm{LET}_{r}$ for the three axial directions can be seen in figure 1. Included are also the two measurements from the Pirs docking module, with and without additional PE shielding. The particle flux $(\Phi)$ and the total $\mathrm{LET}_{r}$ and the heavy-ion component for all data sets are presented in table 2 . The contribution from the trapped component in $\hat{z}$ is visible when looking at the flux and LET rates for all particles in table 2, where we see the highest rates. As expected, this is not the case for the heavy-ion component.

A similar heavy-ion anisotropy has previously been reported by the ALTEA collaboration [6], where data showed a difference in LET $r$ by a factor of 2-3 in the US Destiny module, when comparing the average rates over the orbit for measurements in the three orthogonal directions in the ISS frame of reference.

In figure 2 the Alteino LET spectrum in water is presented for data set 906 together with a PADLES spectrum, with and without PE shielding. Included are also the spectra from the Destiny module measured by DOSTEL in 2001 [2], and ALTEA in 2009 [6]. The difference between the spectra, not counting DOSTEL, mainly arises from the trigger of Alteino and ALTEA which to a large extent makes both detectors less sensitive to low kinetic energy and light nuclei events. Overall ALTEA and Alteino are in agreement. However, the difference around the iron peak $(\approx 150 \mathrm{keV} / \mu \mathrm{m})$ is most likely due to the difference in total shielding between Pirs and Zvezda. The difference with the DOSTEL data is likely a combination of trigger sensitivity and location. The solar particle event which took place during the DOSTEL measurement (15 April 2001) could also be a contributing factor to the difference in the measured flux.

During the course of the ALTCRISS project the ISS average altitude varied by approximately $15 \mathrm{~km}$. The difference in the radiation environment is mostly affected by the trapped component. The heavy-ion component should not be notably affected by such small average altitude changes.

In addition, as stated earlier, solar activity will affect the incoming flux. This should be taken into account when comparing LET rates measured at different times. According to the neutron monitor in Oulu, Finland [28, 29], the field potential parameter for data sets $907(\hat{y})$, $918(\hat{x})$, and $930(\hat{z})$ were 433,390 , and $356 \mathrm{MV}$ respectively. If one would take solar modulation compensation into account, using the spherical approximations model [30] the 
Table 3. Dose and dose equivalent rates from the PADLES pouches.

\begin{tabular}{lccc}
\hline $\begin{array}{l}\text { PADLES } \\
\text { Configuration }\end{array}$ & $\begin{array}{c}\mathrm{D}(\mathrm{TLD}+\mathrm{CR}-39) \\
{\left[\mu \mathrm{Gy} \mathrm{d}^{-1}\right]}\end{array}$ & $\begin{array}{c}\mathrm{H}(\mathrm{TLD}+\mathrm{CR}-39) \\
{\left[\mu \mathrm{Sy} \mathrm{d}^{-1}\right]}\end{array}$ & $\hat{Q}$ \\
\hline Shielded & $269 \pm 16$ & $694 \pm 53$ & $2.58 \pm 0.25$ \\
Shielded & $265 \pm 16$ & $560 \pm 41$ & $2.12 \pm 0.20$ \\
Non-shielded & $281 \pm 17$ & $743 \pm 55$ & $2.65 \pm 0.26$ \\
Non-shielded & $279 \pm 17$ & $686 \pm 53$ & $2.45 \pm 0.14$ \\
\hline
\end{tabular}

Table 4. Dose rate from DLR-TLDs. TLD600 is sensitive to neutron radiation.

\begin{tabular}{lcc}
\hline Configuration & $\begin{array}{c}\text { TLD600 } \\
{\left[\mu \mathrm{Gy} \mathrm{d}^{-1}\right]}\end{array}$ & $\begin{array}{c}\text { TLD700 } \\
{\left[\mu \mathrm{Gy} \mathrm{d}^{-1}\right]}\end{array}$ \\
\hline Shielded & $224 \pm 8$ & $206 \pm 7$ \\
Shielded & $234 \pm 7$ & $206 \pm 4$ \\
Non-shielded & $262 \pm 7$ & $229 \pm 4$ \\
\hline
\end{tabular}

compensated LET rate ratios would be even higher, thus implying the non-compensated heavy-ion LET rate ratios of $15 \pm 2$ for $\hat{y} / \hat{x}$ and $1.7 \pm 0.2$ for $\hat{y} / \hat{z}$ are to be considered conservative.

When comparing flux rates in data sets 902 and 903 we can see a reduction by about $25 \%$ when the polyethylene was added. The situation is similar in Zvezda $\hat{x}$ direction (925 versus 924), as well as for 910 and 930 in $\hat{z}$. However, both measurement in $\hat{z}$ are, according to documentation with the additional shield mounted. This is also the case for the $\hat{y}$ measurements, where all rates are in agreement. Solar modulation effects should only increase the difference in flux rate. The only explanation we currently can provide is that there either was a typographic error in the documentation, or that during data set 930 some additional equipment was accidentally placed in front of the detector, thus adding to the shielding.

The dose, dose equivalent, and average $Q$-value for Alteino are also presented in table 2 . The corresponding values measured by the passive detectors are presented in tables 3 and 4 for PADLES and DLR-TLDs respectively. PADLES combines the dose measurements for $<10 \mathrm{keV} / \mu \mathrm{m}$ from the TLDs with $\geqq 10 \mathrm{keV} / \mu \mathrm{m}$ range from the CR-39. The large energy range obtained when combining TLDs and CR-39 detectors enables measurement of the dose contribution down to $\sim 0.1 \mathrm{keV} / \mu \mathrm{m}$. By taking the ratio between the passive measurements and Alteino we obtain an estimate of the dose contribution by low kinetic energy and light nuclei. The data should primarily be compared with Alteino data sets 902 and 903, given acquisition time and detector location. The dose rate ratio when compared with average PADLES values amounts to a factor of $3.9 \pm 0.2$ lower for Alteino for the non shielded measurements and $4.7 \pm 0.3$ for the shielded measurements. The corresponding values for the DLR-TLDs are 3.2 and 3.6 for the TLD700 and 3.7 and 3.9 for the TLD600. This would imply that the contribution to the dose by low kinetic energy and light nuclei can be as high as about $75 \%$.

\section{Conclusions}

In this work we have presented data on particle flux, LET, dose, and dose equivalent rates from various locations inside the Russian section of the ISS. In addition we report on dose 
and dose equivalent rates by passive detectors, measured in conjunction with Alteino. When LET spectrum from the Zvezda service module was compared to spectra from the Destiny module measured by the ALTEA detector we see good agreement although some variations in flux. We attribute these variations primarily to the difference in total shielding (hull and rack configuration combined) at the different measuring locations and detector triggering sensitivity. When comparing Alteino LET rate in the three axial directions we see a clear anisotropy for heavy-ions, where the LET rate in $\hat{y}$ is about a factor 10 higher, and in $\hat{z} 9$ times higher rate than in $\hat{x}$. Such a heavy-ion anisotropy has earlier been measured by ALTEA [6]. The dose and dose equivalent tell a similar story; ranging from $25-40 \mu \mathrm{Gy} \mathrm{d}^{-1}$ in $\hat{x}$, to about $50-75 \mu \mathrm{Gy} \mathrm{d}^{-1}$ in $\hat{z}$, and around $60 \mu \mathrm{Gy} \mathrm{d}^{-1}$ in $\hat{y}$. However, for the dose equivalent there is a higher difference in $\hat{y}$ and $\hat{z}$ : about $470 \mu \mathrm{Sv} \mathrm{d}^{-1}$ and $300-350 \mu \mathrm{Sv} \mathrm{d}^{-1}$ respectively and $50-110 \mu \mathrm{Sv} \mathrm{d}{ }^{-1}$ in $\hat{x}$ with an average $Q-$ value ranging from about 2 to 8 . The statistical errors are less than $1 \%$.

For the passive dosimeters, the PADLES (TLD+CR-39) measures a shielded dose rate of about $269 \pm 16 \mu \mathrm{Gy} \mathrm{d}^{-1}$ and a dose equivalent of $694 \pm 53 \mu \mathrm{Sv} \mathrm{d}^{-1}$. For the non-shielded measurements the values peaked at $281 \pm 17 \mu \mathrm{Gy} \mathrm{d}^{-1}$ and $743 \pm 55 \mu \mathrm{Sv} \mathrm{d}^{-1}$. The DLRsupplied TLD700 measures about $200 \mu \mathrm{Gy} \mathrm{d}^{-1}$ for the shielded configuration and the nonshielded $230 \mu \mathrm{Gy} \mathrm{d}^{-1}$ and for the neutron sensitive TLD600 the rates are about $10 \%$ higher.

The contribution to the dose rate from low kinetic energy particles and light nuclei is about $75 \%$, whereas the corresponding contribution to dose equivalent is about $40 \%$ depending on the shielding configuration.

\section{Acknowledgments}

The ALTCRISS project was supported by ESA. We would also like to extend our gratitude towards Riken, ACCC-Riken, the Russian Space Agency, to the Italian Space Agency (ASI 2013-034-R.0 and ALTEA-MoMa contract), to INFN (Instituto Nazionale di Fisica Nucleare), and the Swedish National Space Board (SNSB) for financial support, building the detector, launching it to the Space Station, and for data handling and analysis. We thank all colleagues and friends at MARS center, Napoli, for the fundamental support and help for the whole duration of the mission.

\section{References}

[1] Durante M and Cucinotta F A Nov 2011 Physical basis of radiation protection in space travel Rev. Mod. Phys. 83 1245-81

[2] Reitz G, Beaujean R, Benton E, Burmeister S, Dachev Ts, Deme S, Luszik-Bhadra M and Olko P 2005 Space radiation measurements on-board iss-the dosmap experiment Radiat. Prot. Dosim. 116 374-9

[3] Nagamatsu A, Murakami K, Kitajo K, Shimada K, Kumagai H and Tawara H 2013 Area radiation monitoring on iss increments 17 to 22 using padles in the Japanese experiment module kibo Radiat. Meas. 59 84-93

[4] Wilson J W et al 2006 International Space Station: a testbed for experimental and computational dosimetry Adv. Space Res. 37 1656-63

[5] Narici L, Casolino M, di Fino L, Larosa M, Larsson O, Picozza P and Zaconte V 2012 Iron flux inside the International Space Station is measured to be lower than predicted Radiat. Meas. 47 $1030-4$

[6] di Fino L, Casolino M, de Santis C, Larosa M, la Tessa C, Narici L, Picozza P and Zaconte V 2011 Heavy-ion anisotropy measured by altea in the International Space Station Radiat. Res. 176 397-406 
[7] Zaconte V, Casolino M, di Fino L, la Tessa C, Larosa M, Narici L and Picozza P 2010 High energy radiation fluences in the ISS-USLab: ion discrimination and particle abundances Radiat. Meas. $45168-172$

[8] La Tessa C, Di Fino L, Larosa M, Narici L, Picozza P and Zaconte V 2009 Estimate of the space station shielding thickness at a USLab site using altea measurements and fragmentation cross sections Nucl. Instrum. Methods Phys. Res. B 267 3383-7

[9] Casolino M et al 2003 Study of the radiation environment on MIR space station with Sileye-2 experiment Adv. Space Res. 31 135-40

[10] Bidoli V et al 2000 Study of cosmic rays and light flashes on board space station MIR: the Sileye experiment Adv. Space Res. 25 2075-9

[11] Fuglesang C 2007 Using the human eye to image space radiation or the history and status of the light flash phenomena Nucl. Instrum. Methods Phys. Res. A 580 861-5

[12] Avdeev S et al 2002 Eye light flashes on the mir space station Acta Astronaut. 50 511-25

[13] Narici L 2008 Heavy ions light flashes and brain functions: recent observations at accelerators and in spaceflight New J. Phys. 10075010

[14] Casolino M et al 2007 The altcriss project on board the International Space Station Adv. Space Res. 40 1746-53

[15] Casolino M et al 2002 The sileye-alteino experiment on board the International Space Station Nucl. Phys. B 113 71-78

[16] Casolino M et al 2006 Detector response and calibration of the cosmic-ray detector of the Sileye-3/ Alteino experiment Adv. Space Res. 37 1691-6

[17] Casolino M et al 2006 Relative nuclear abundances inside ISS with Sileye-3/Alteino experiment Adv. Space Res. 37 1685-90

[18] Cameron J R, Suntharalingam N and Kenney G N 1968 Thermoluminescent dosimetry

[19] Cartwright B, Shirk E and Price P 1978 A nuclear-track-recording polymer of unique sensitivity and resolution Nucl. Instrum. Methods 153 457-60

[20] Nagamatsu A, Masukawa M, Kamigaichi S, Kumagai H, Masaki M, Yasuda N, Yasuda H, Benton E, Hayashi T and Tawara H 2006 Development of the space radiation dosimetry system PADLES Proc. 20th Workshop on Radiation Detectors and Their Uses, KEK Proc. (Tsukuba) vol 726

[21] Ohnishi T, Takahashi A, Nagamatsu A, Omori K, Suzuki H, Shimazu T and Ishioka N 2009 Detection of space radiation-induced double strand breaks as a track in cell nucleus Biochem. Biophys. Res. Commun. 390 485-8

[22] Larsson O et al 2014 Relative nuclear abundance from $\mathrm{C}$ to $\mathrm{Fe}$ and integrated flux inside the russian part of the iss with the Sileye-3/Alteino experiment J. Phys. G: Nucl. Part. Phys. 41 015202

[23] Sullivan J D 1971 Geometric factor and directional response of single and multi-element particle telescopes Nucl. Instrum. Methods 95 5-11

[24] Agostinelli S et al 2003 Geant4-a simulation toolkit Nucl. Instrum. Methods Phys. Res. A 506 250-303

[25] Allison J et al 2006 Geant4 developments and applications IEEE Trans. Nucl. Sci. 53 270-8

[26] Benton E, Benton E and Frank A 2010 Conversion between different forms of let Radiat. Meas. 45 957-9

[27] Valentin J 2003 Relative biological effectiveness (rbe), quality factor (q), and radiation weighting factor (wr): Icrp publication 92 Ann. ICRP $331-121$

[28] Usoskin I G, Alanko-Huotari K, Kovaltsov G A and Mursula K 2005 Heliospheric modulation of cosmic rays: Monthly reconstruction for 1951-2004 J. Geophys. Res. 110 A12108

[29] Usoskin I G, Bazilevskaya G A and Kovaltsov G A 2011 Solar modulation parameter for cosmic rays since 1936 reconstructed from groundbased neutron monitors and ionization chambers J. Geophys. Res. 116 A02104

[30] Gleeson L J and Axford W I 1968 Solar modulation of galactic cosmic rays Astrophys. J. 154 $1011-26$ 